\title{
100. Namenstag des DZK
}

\author{
100th Anniversary of the Name DZK \\ (German Central Committee against Tuberculosis)
}

Autor

Institut
R. Loddenkemper

Deutsches Zentralkomitee zur Bekämpfung der Tuberkulose (DZK), Berlin
Bibliografie

DOI $10.1055 / \mathrm{s}-2006-944262$ Pneumologie 2006; 60; 560-561

(c) Georg Thieme Verlag KG

Stuttgart · New York

ISSN 0934-8387

Korrespondenzadresse

Prof. Dr. Robert

Loddenkemper

Generalsekretär des DZK

Lungenklinik Heckeshorn

HELIOS Klinikum

Emil von Behring

Zum Heckeshorn 33

14109 Berlin

loddheck@zedat.fu-berlin.de

Serienherausgeber

R. Kropp, Fulda
Am 31. Mai 1906 wurde das 1895 gegründete „Deutsche Central Komitee zur Errichtung von Heilstätten für Lungenkrankheiten“ umbenannt in „Deutsches Zentralkomitee zur Bekämpfung der Tuberkulose“ [1]. Diese Bezeichnung, die leider etwas umständlich klingt, ist auch noch heute gültig.

Zur ursprünglichen Namensgebung sei daran erinnert, dass zu dieser Zeit die Heilstättenbewegung en vogue war. Sie ging auf Hermann Brehmer zurück und seine sich später als falsch erweisende Therapie von „immunen Orten“, an denen es keine Schwindsüchtigen gab. Er folgerte daraus, dass sich an diesen „immunen Orten“ eine bestehende Tuberkulose besser rückbilden bzw. sogar ausheilen lasse. So gründete er das erste Tuberkulose-Sanatorium in Görbersdorf (heute Sokolov) im schlesischen Riesengebirge im Jahr 1856, also vor 150 Jahren [1,2]. 1863 baute er es aus [3], heute wirkt es in seinem roten Backsteinstil mit zahlreichen Türmchen wie ein verfallenes Zauberschloss.

Den Siegeszug der Heilstättenbewegung erlebte Brehmer nicht mehr. Nachdem sein früherer Patient und Schüler Peter Dettweiler in Falkenstein/ Taunus 1876 ein Sanatorium mit der Einführung der Freiluft-Liegekur als Basis der TuberkuloseTherapie gegründet hatte [4], breitete sich die Sanatoriums-Idee in Deutschland, Europa und Nordamerika aus $[5,6]$.

Die Tuberkulose war in dieser Zeit eine wirkliche Volkskrankheit und eine der Haupttodesursachen in den Industrieländern. Besonders betroffen waren die mittleren, im Arbeitsleben besonders aktiven Jahrgänge sowie die sozial schwachen Gesellschaftsschichten, die zum Teil unter miserablen hygienischen Bedingungen lebten $[2,3]$. Nach dem Scheitern von Robert Kochs Gedanken, das Tuberkulin als Heilmittel gegen die Tuberkulose anzuwenden, und wegen des Fehlens anderer therapeutischer Möglichkeiten - lediglich die Kollapstherapie und chirurgische
Maßnahmen kamen in Einzelfällen in Betracht $[7,8]$ - wurde großer Wert auf die Gründung von Lungenheilstätten, meist außerhalb der Großstädte, gelegt.

Aus Schottland kam dann über Frankreich und Belgien jedoch die Erkenntnis nach Deutschland, wie wichtig Fürsorgemaßnahmen für die Bekämpfung der Tuberkulose sind [1]. Um sich auch diesen Aufgaben zu widmen, erfolgte die Umbenennung in „Deutsches Zentralkomitee zur Bekämpfung der Tuberkulose“. Es hatte neben der Errichtung von Heilstätten für Lungenkranke als Ziele die wirtschaftliche und hygienische Fürsorge für die Lungenkranken, die Förderung von Wissenschaft und Forschung auf dem Gebiet der Tuberkulose und die Aufklärung der Bevölkerung („Volksaufklärung“). Ehrenpräsident des Komitees war der Reichskanzler, Präsident der jeweilige Minister oder Staatssekretär des Inneren, und das DZK war Ratgeber der Reichsregierung in allen Fragen der Tuberkulose [1].

Durch die Initiative seines ersten Generalsekretärs und eigentlichen Gründers, Gottfried von Pannwitz, gelang es, den ersten großen wissenschaftlichen „Kongress zur Bekämpfung der Tuberkulose als Volkskrankheit" 1899 nach Berlin $\mathrm{zu}$ berufen. $\mathrm{Zu}$ diesem Kongress kamen über 2000 Teilnehmer, darunter 200 Mitglieder ausländischer Regierungen. Die Konferenz wuchs damit weit über den ursprünglich vorgesehenen Rahmen hinaus und wurde zur ersten internationalen Tuberkulosekonferenz überhaupt [1]. Anlässlich dieser ersten großen Tuberkulosetagung wurde ein „Internationales Zentralbüro zur Bekämpfung der Tuberkulose“ mit dem Sitz in Berlin gegründet. Dieses Büro war der Vorläufer der heutigen „Internationalen Union gegen Tuberkulose und Lungenkrankheiten“ (IUATLD). 1902 wurde durch das Büro die „I. Internationale Tuberkulosekonferenz" vom 22. bis 26. Oktober in Berlin ausgerichtet [1]. 
Die Betonung des Fürsorgewesens veranlasste 1913 die Gründung des „Tuberkulosefürsorgeblatt“, das später in „Reichstuberkuloseblatt“ umbenannt wurde [1]. 1946 wurde die Zeitschrift „Der Tuberkulosearzt“ als Nachfolgeorgan gegründet. Diese Zeitschrift wurde jeweils der aktuellen Entwicklung unseres Fachgebiets angepasst, mehrfach in ihrem Namen verändert und führt schließlich seit 1988 den Namen „Pneumologie“.

Heute besteht eine enge Zusammenarbeit des DZK mit der Deutschen Gesellschaft für Pneumologie und Beatmungsmedizin. Der Präsident des DZK ist laut Mitgliederbeschluss 1985 automatisch der jeweilige, alle zwei Jahre wechselnde Präsident der DGP.

Nach wie vor hat das DZK die Rechtsform eines „gemeinnützigen Vereins“ mit ausschließlich juristischen Personen als Mitgliedern. Durch das politische Gewicht der Mitglieder und durch das wissenschaftliche Gewicht der Arbeitsgruppen des DZK ist dieses ein wichtiger Meinungs- und Entscheidungsträger in allen Tuberkulosefragen der Bundesrepublik. Einer der Tätigkeitsschwerpunkte des DZK ist neben der Öffentlichkeitsarbeit und der Aufklärungs- und Beratungstätigkeit die Aufrechterhaltung der Kontakte zu internationalen Organisationen, die hinsichtlich der Tuberkulosekontrolle eine bedeutende Rolle haben. Die jährlich erscheinenden Informationsberichte geben jeweils eine Übersicht über die aktuelle Tuberkulosesituation in Deutschland, die Entwicklung der weltweiten Tuberkulose-Epidemiologie und ihre möglichen Auswirkungen auf Deutschland [9]. Daneben werden für die Fürsorge, Praxis und Klinik wichtige wissenschaftliche Entwicklungen in Expertisen aufgezeigt [10]. Diese gehen dann auch in die regelmäßig aktualisierten Empfehlungen des DZK ein, die von nationalen und internationalen Experten erarbeitet werden [11 - 13].

Die DZK-Studie zur Epidemiologie der Tuberkulose in Deutschland 1996-2000, die unter der Leitung von Dr. M. Forßbohm in Zusammenarbeit mit dem ÖGD und mit Förderung durch das BMG durchgeführt wurde, hatte großen Einfluss auf die Ausgestaltung des Infektionsschutzgesetzes [15], welches am 1. Januar 2001 das Bundesseuchengesetz ablöste [16]. Seither erscheinen die epidemiologischen Daten nicht mehr im DZK-Jahresbericht, sondern in den RKI-Jahresberichten, zum ersten Mal mit den Daten des Jahres 2001 [17]. Mit der gerade beendeten DZK-Studie zur molekularen Epidemiologie der Tuberkulose, ebenfalls mit Unterstützung des Bundesministeriums für Gesundheit durchgeführt, sind Argumente für eine flächendeckende Einführung dieser neuen epidemiologischen Verfahren gesammelt worden [18].

Die Arbeit des DZK behält angesichts der sich global immer noch verschlechternden Tuberkulosesituation und der zunehmenden Medikamentenresistenzen bei den Migranten, die Deutschland erheblich betreffen, auch deshalb große Bedeutung, weil es in Deutschland im Vergleich zu anderen Industriestaaten nach wie vor im Bereich der Lungenkrankheiten wenige Einrichtun- gen für Forschung und Lehre gibt. Die Aufrechterhaltung der Kontakte zu Institutionen/Organisationen, die hinsichtlich der Tuberkulosekontrolle eine bedeutende Rolle, sowohl national als auch international, spielen, gehört ebenfalls zu den wesentlichen Aufgaben des DZK $[1,9]$.

\section{Literatur}

1 Ferlinz R. Die Tuberkulose in Deutschland und das Deutsche Zentralkomitee zur Bekämpfung der Tuberkulose. Pneumologie 1995; 49: $617-632$

2 Voigt J. Zur Sozialgeschichte der Tuberkulose. Verlorene Siege. In: Konietzko N (Hrsg.). 100 Jahre Deutsches Zentralkomitee zur Bekämpfung der Tuberkulose (DZK). Der Kampf gegen die Tuberkulose. pmiVerlagsgruppe, 1996: $51-75$

3 Hähner-Rombach S. Die Freiluft-Liegekur. In: Sozialgeschichte der Tuberkulose. Vom Kaiserreich bis zum Ende des Zweiten Weltkriegs unter besonderer Berücksichtigung Württembergs. Stuttgart: Franz Steiner Verlag, 2000: $144-144$

4 Tracht D. Architektur von Volksheilstätten um 1900. Pneumologie 2005; 59: $901-909$

5 Chrétien J. Le Sanatorium. In: La tuberculose. Parcours imagé. Band I. Propos. Hauts-de-France. Editions, 1995: 75-94

6 Dubos R, Dubos J. XIII. Healthy Living and Sanatoria. In: The White Plagne. Tuberculosis, Man, and Society. Boston: Little, Brown and Company, 1952: $173-181$

7 Jacobaeus HC. Endopleurale Operationen unter Leitung des Thorakoskops. Beitr Klin Tuberk 1916; 35: 1 - 15

8 Sauerbruch F. Die historische Entwicklung der operativen Behandlung der Lungentuberkulose. Zs f Tuberkulose 1930; 57: 289-294

9 Deutsches Zentralkomitee zur Bekämpfung der Tuberkulose. 29. Informationsbericht. Berlin: DZK, 2006

10 Hauer B, Loddenkemper $R$, Detjen A et al. Interferon- $\gamma$-Tests in der Tuberkulose-Diagnostik - Aktueller Stand. Pneumologie 2006; 60: 29 44

11 Schaberg T, Hauer B, Haas W et al. Latente tuberkulöse Infektion: Empfehlungen zur präventiven Therapie bei Erwachsenen in Deutschland. Pneumologie 2004; 58: 255-270

12 Schaberg T, Hauer B, Loddenkemper R et al. Empfehlungen zur Anwendung von Atemschutzmasken bei Tuberkulose. Pneumologie 2004; 58: $92-102$

13 Schaberg T, Forßbohm M, Hauer B et al. Richtlinien zur medikamentösen Behandlung der Tuberkulose im Erwachsenen- und Kindesalter. Pneumologie 2001; 55: $494-511$

14 Forßbohm M. Studie des DZK zur Epidemiologie der Tuberkulose. Abschlussbericht 1996-2000 (26333 Fälle). In: Deutsches Zentralkomitee zur Bekämpfung der Tuberkulose. 28. Informationsbericht. Berlin: DZK, 2004

15 Gesetz zur Verhütung und Bekämpfung von Infektionskrankheiten beim Menschen (IfSG). Bundesgesetzblatt I, 2000: 1045 - 1045

16 Forßbohm M, Loytved G. Auswirkungen des Infektionsschutzgesetzes auf die Erfassung der Tuberkulose: Meldepflicht, Falldefinition, Datenerhebung und -übermittlung. In: Deutsches Zentralkomitee zur Bekämpfung der Tuberkulose. 28. Informationsbericht. Berlin: DZK, 2004

17 Robert Koch-Institut. Bericht zur Epidemiologie der Tuberkulose in Deutschland für 2001. Berlin: Mercedes Druck, 2003

18 Deutsches Zentralkomitee zur Bekämpfung der Tuberkulose. Studie des DZK: Untersuchungen zur Tuberkulose in Deutschland: Molekulare Epidemiologie, Resistenzsituation und Behandlung. Abschlussbericht (überarbeitete Fassung). Berlin: DZK, 2006 